\section{Explanations for} aberrations

\author{
K.W. Jones
}

Cancer Cytogenetics. By Sverre Heim and Felix Mitelman. Alan R. Liss, New York Wiley, Chichester: 1987. Pp. 309. \$35, $£ 27.50$.

FOR many years, the study of chromosomes of tumours seemed to offer little insight into the causation of cancer and attracted comparatively little general interest. Things changed with the original finding of the $\mathrm{Ph} 1 \mathrm{marker}$ chromosome in chronic myeloid leukaemia by Nowell and Hungerford in 1961. Since then, improvements in cytological techniques and the discovery of viral oncogenes and cellular proto-oncogenes has made cancer cytogenetics a focal discipline for molecular biologists, virologists, geneticists, pathologists and clinicians.

Given the multiplicity of contributing subjects, and the growing appreciation of the complexities underlying the pathogenesis of cancer, this is a difficult field to encompass in a single book. Heim and Mitelman have nonetheless succeeded in producing a readable, critical and up-todate account, which not only conveys both historical perspective and the current excitement in this area, but which will be a valuable reference source.

The book emphasizes both the successes and limitations of cancer cytogenetics, for example the fact that most tumours have remained refractory to cytogenetic analysis so that 87 per cent of analysable cancers are leukaemias. Unfortunately, the intractable class includes carcinomas which are quantitatively the most important. Moreover, as the authors point out, genetic changes of as much as $10^{6}-10^{7}$ base pairs may occur without there being a visible cytogenetic consequence, so that chromosome studies, although invaluable, reveal just the tip of what might be a very large iceberg.

Nevertheless, the striking fact which has emerged is that chromosomal aberrations in human cancer are not random but are clustered in approximately 83 chromosomal bands; at least one of these particular regions, apparently, is involved in 96 per cent of neoplasms. Some of these are known fragile sites and/or correspond to the locations of known proto-oncogenes, which perhaps explains why changes in these abnormal chromosome patterns frequently have clinical significance both as to treatment and prognosis. Interestingly, the precise character of abnormalities also appears to show geographical variation in apparently identical malignancies, suggesting differences in relevant gene frequencies or in exposure to

oncogenic factors

Cancer Cytogenetics is as much a clinical manual as an academic text, with specific sections in each chapter summarizing the clinical correlations for various cancers, as one would expect from two distinguished clinical geneticists. But because this material is skilfully integrated with accounts of the underlying molecular mechanisms, where such information exists, the book will draw molecular biologists and clinicians into a closer mutual understanding, which, I suspect, was the authors' intention. The multifaceted approach to the subject matter constantly throws up stimulating questions and means the reader's interest is held throughout what, in other hands, could easily have been a dull book. The authors are not reticent in putting forward

\section{Versatile vectors}

\section{Tim Harris}

Vectors: A Survey of Molecular Cloning Vectors and Their Uses. Edited by Raymond L. Rodriguez and David $\mathrm{T}$. Denhardt. Butterworths:1988. Pp.578. $£ 45, \$ 54.95$

ONCE it was that one obtained a vector for cloning DNA from a friend or colleague. These days, as molecular biology kits (and molecular biologists) proliferate, many vectors can be bought off the shelf, tailormade for a specific task, be it cDNA or genomic cloning, transcription or expression, or for introduction into animal or plant cells. The diversity and usefulness of modern vectors can be appreciated from this collection of multi-author reviews, although, inevitably, some recent advances have not been included.

Balance is all-important in a book of this kind; to some extent judgement over which topics should have been covered in more detail and which in less detail is a matter of individual preference. For me, the editors have not got it quite right. The emphasis is on bacteria, with at least twothirds of the chapters dealing with both the gram negative and gram positive types. General topics include a useful potted history and genealogy of pBR322, and descriptions of $\mathrm{M} 13$ and lambda phages, cosmids and phagemids all at a level suitable for third-year undergraduates. More specific coverage of plasmids for cloning promoters and terminators, SP6 and T7 promoter-directed transcription, copy number control and for cloning into broad host ranges is also provided in this section. There is the notable absence, however, of a chapter dedicated to sitedirected mutagenesis - an increasingly important technique which is only referred to in passing, in amongst the their own views, but fact, uncertainty, hypothesis and opinion are carefully and consistently differentiated, which makes the volume of particular value to students.

In all, this is a stimulating and lucid book. It contains some 14 tables, which condense and classify a large amount of useful information, 63 figures and diagrams, and over 600 references, many of which are to papers published in 1987. It will appeal to oncologists and molecular biologists actively engaged in research, as well as to pathologists, clinicians, teachers and students. Given its wide appeal, in my view no science library can afford not to have several copies of it.

$K . W$. Jones is a Reader in the Department of Genetics, University of Edinburgh, King's Buildings, West Mains Road, Edinburgh EH9 $3 J N, U K$.

filamentous phages.

The remainder of the book is concerned with vectors for other systems, including fungal cells, insects and insect cells, and animal and plant cells. It is not quite fair to say that these chapters seem to have been added as an afterthought, but there is a suspicion of it - quite a surprise considering the importance of the subject matter for molecular biology in general and biotechnology in particular. Mammalian cell vectors are particularly poorly dealt with, little attempt being made to evaluate critically the relative merits of one system versus another, and there is little mention of the vectors being used to produce large amounts of proteins for therapy (for example t-PA, erythropoeitin and various antibodies).

It is a pity that an industrial viewpoint is not given here, as it is in one of the two chapters concerned with the transformation of plant cells. A similar criticism can be levelled at the section on yeast, which although it covers the different types of shuttle plasmid available, does not go into the details of those used to express foreign genes such as hepatitis B surface antigen or $\alpha 1$-antitrypsin. While application is not the be all and end all, a lot of people do use vectors for expression purposes.

So, like the curate's egg, the book is good in parts. If bacterial vectors are what you want to know about, then it will be pretty useful, although the quality of the diagrams in general leaves much to be desired. For other cells it would be better to go to the excellent, up-to-date and relevant reviews that are available.

Tim Harris is Head of Molecular Biology at Celltech Ltd, 216 Bath Road, Slough SL1 4EN, $U K$.

\section{New in Britain}

Invisible Frontiers: The Race to Synthesize a Human Gene, by Stephen S. Hall, is now available in Britain. Publisher is Sidgwick and Jackson, price is $£ 13.95$. For review see Nature 329, 399 (1987). 